\title{
Interactions between Caenorhabditis elegans Individuals during Chemotactic Response
}

\author{
Tetsuya Matsuura*, Taketo Sato and Ryuzo Shingai \\ Department of Welfare Engineering, Faculty of Engineering, \\ Iwate University, Morioka 020-8551, Japan
}

\begin{abstract}
The chemotactic response of the nematode Caenorhabditis elegans is known to be affected by the population density on an assay plate, suggesting the existence of interactions between individual animals. To clarify the interactions between individuals during chemotaxis, we investigated the effect of population density at an attractant area on the chemotactic response to water-soluble sodium acetate and odorant diacetyl using wild-type N2 animals and daf-22 (m130) mutants, which have defective pheromone secretion but can sense pheromone. The chemotaxis index of N2 animals at $90 \mathrm{~min}$ of the assay negatively correlated with the number of animals on the assay plate regardless of the type of attractant used $(p<0.01)$. On the other hand, there was no significant difference in the chemotaxis indices of daf-22 (m130) mutants for either of the attractants between the low- and high-population groups. When daf-22 (m130) mutants of a high population density were placed at the attractant location in advance, the chemotaxis index of N2 animals was almost the same as that in the control assay in which no animals were placed at the attractant location in advance. When N2 animals of a high population density were placed at the attractant location in advance, the chemotaxis indices of $\mathrm{N} 2$ animals and daf-22 (m130) mutants were significantly smaller than those obtained in the control assay $(p<0.05)$. In the absence of an attractant, we observed a decline in the fraction of animals in the neighborhood of $\mathrm{N} 2$ animals of a high population density, although the nematodes were not influenced by daf-22 (m130) mutants of a high population density. These results suggest that the attraction of nematodes to chemicals is inhibited by an increase in the concentration of the pheromone generated by $\mathrm{N} 2$ animals at the attractant location.
\end{abstract}

Key words: Caenorhabditis elegans, chemotaxis, diacetyl, pheromone, population density, sodium acetate

\section{INTRODUCTION}

The nematode, Caenorhabditis elegans, lives in soil and at air and water interfaces, and feed on soil bacteria (Andrew and Nicholas, 1976). It shows a chemotactic response to various compounds including some ions (e.g., $\mathrm{Na}^{+}$and $\mathrm{Cl}^{-}$), amino acids, alcohols and other substances, which are produced by food bacteria and act as natural attractants of chemotaxis (Ward, 1973; Bargmann and Horvitz, 1991; Bargmann et al., 1993). The presentation of chemical stimuli such as water-soluble substrates (e.g., $\mathrm{NaCl}$ and lysine) and volatile odorants (e.g., diacetyl and isoamyl alcohol) elicits an attractive response of the nematode (Ward, 1973; Dusenbery, 1974; Bargmann et al., 1993), and presentation of chemical stimuli such as $\mathrm{Cu}^{2+}$, SDS and benzaldehyde at high concentrations elicits an

\footnotetext{
${ }^{*}$ Corresponding author. Phone: +81-19-621-6376;

Fax : +81-19-621-6376;

E-mail: matsuura@iwate-u.ac.jp
}

avoidance response (Bargmann et al., 1993; Nuttly et al., 2001; Hilliard et al., 2002). These chemicals are sensed by chemosensory neurons in the head sensory organ amphid and the tail organ phasmid (Ward, 1973; Bargmann and Horvitz, 1991; Hilliard et al., 2002), and information on these chemicals is transmitted to several interneurons and play a significant role in mediating chemotactic behavior.

In chemotaxis assays for nematodes, many animals are generally used in one assay plate to evaluate the fraction of animals that gather at an attractant area; this fraction is known as the chemotaxis index (Bargmann et al., 1993; Matsuura et al., 2004a). However, the relationship between the index and population of nematodes on an assay plate was not clarified. Recently, a difference in the time of aversive response to $100 \%$ benzaldehyde between groups and individually tested animals was observed (Nuttly et al., 2001). Moreover, in a preliminary report, we showed decreases in chemotaxis indices for sodium acetate and diacetyl with an increase in the number of wild-type animals on an 
assay plate (Matsuura et al., 2004b). Although these observations indicate the existence of interactions between individuals during population assays, the regulation mechanism of chemotactic response of nematodes has not yet been clarified.

It is known that the dauer pheromone is produced by all nematodes, and its concentration is used by these animals as a measure of population density (Golden and Riddle, 1984). C. elegans exhibits developmental selection in its life cycle which is influenced by environmental conditions. Under favorable conditions, its life cycle consists of four larval stages. If environmental conditions are unfavorable, nematodes may have arrested development following the L2 stage and become a dauer larva which is well adapted for long-term survival and is tolerant to harsh conditions (Riddle and Albert, 1997). The selection between development into normal and dauer larvae is modulated by food availability, temperature and dauer pheromone concentration (Riddle and Albert, 1997; Ailion and Thomas, 2000). It is possible that an increase in pheromone concentration with population density affects the behavior of $C$. elegans during chemotaxis.

The aim of the present study was to investigate the effect of population density on the chemotactic response of nematodes and to clarify the mechanism of interactions between individual animals. First, we examined the chemotactic response of wild-type N2 animals and daf-22 (m130) mutants, which were defective in pheromone secretion (Golden and Riddle, 1985), to water-soluble sodium acetate and odorant diacetyl under the condition of various populations. Then, we investigated the chemotactic responses of the nematodes under the condition in which N2 animals or daf-22 (m130) mutants of a high population densities were placed at the attractant location in advance to clarify the effect of pheromone on chemotactic response. The effect of pheromone on the animal's behavior was also examined in the absence of an attractant. The data of the present study may possibly lead to a better understanding of the principle of communication between nematodes.

\section{MATERIALS AND METHODS}

\section{Animals}

Caenorhabditis elegans wild-type strain (Bristol N2) and daf22(m130) mutants, which were defective in pheromone secretion, were obtained from the Caenorhabditis Genetics Center at the University of Minnesota. The nematodes were grown and maintained on NGM agar plates $(3 \mathrm{~g} / \mathrm{l} \mathrm{NaCl}, 2.5 \mathrm{~g} / \mathrm{l}$ polypeptone, $5 \mathrm{mg} / \mathrm{l}$ cholesterol, $1 \mathrm{mM} \mathrm{CaCl}_{2}, 1 \mathrm{mM} \mathrm{MgSO}_{4}, 25 \mathrm{mM} \mathrm{KH}_{2} \mathrm{PO}_{4}(\mathrm{pH} \mathrm{6.0)}$ and 17 $\mathrm{g} / \mathrm{l}$ agar) with the Escherichia coli OP50 strain in an incubator (SANYO MIR253) at $20^{\circ} \mathrm{C}$ (Brenner, 1974). In all experiments, we used synchronously staged young adult hermaphrodites of N2 animals obtained approximately $70 \mathrm{hr}$ after hatching at $20^{\circ} \mathrm{C}$. We also used young adult hermaphrodites of daf-22 (m130) mutants obtained approximately $75 \mathrm{hr}$ after hatching at $20^{\circ} \mathrm{C}$, because the development of daf-22 (m130) mutants was more delayed in comparison with that of $\mathrm{N} 2$ animals.

\section{Chemotaxis assays}

In all chemotaxis assays, we used 9-cm-diameter tissue culture dishes for assay containing $1 \mathrm{mM} \mathrm{CaCl}_{2}, 1 \mathrm{mM} \mathrm{MgSO}_{4}, 5 \mathrm{mM}$ $\mathrm{KH}_{2} \mathrm{PO}_{4}(\mathrm{pH} \mathrm{6.0)}$ and $15 \mathrm{~g} / \mathrm{l}$ agarose, in which $\mathrm{NaCl}$ was omitted. The nematode showed the highest attraction to $1.0 \mathrm{M}$ sodium acetate $\left(\mathrm{CH}_{3} \mathrm{COONa}\right)$ and $0.1 \%$ diacetyl in our previous study (Matsuura et al., 2004a). Therefore, we used these concentrations for the attractants in chemotaxis assays. Sodium acetate $(7 \mu \mathrm{l})$ was placed twice on the surface of an assay plate (attractant location; location A in Fig. 1A) 15 18 hr and $3 \mathrm{hr}$ before the start of the experiment to obtain a concentration gradient for the assay of sodium acetate. The assay plate was placed in an incubator at $20^{\circ} \mathrm{C}$. To anesthetize the animals, $1 \mu \mathrm{l}$ of $0.5 \mathrm{M}$ sodium azide was spotted at the same location as that of sodium acetate shortly before the experiment. As a control, $1 \mu \mathrm{l}$ of $0.5 \mathrm{M}$ sodium azide was also spotted $4 \mathrm{~cm}$ away from the attractant location (control location; location B in Fig. 1A). For the assay of diacetyl, $1.5 \mu \mathrm{l}$ of diacetyl dissolved in $99.5 \%$ ethanol and $1 \mu \mathrm{l}$ of $0.5 \mathrm{M}$ sodium azide were spotted at location A (attractant location, Fig. $1 \mathrm{~A}$ ) of an assay plate shortly before the experiment. As a control, $1.5 \mu \mathrm{l}$ of $99.5 \%$ ethanol and $1 \mu \mathrm{l}$ of $0.5 \mathrm{M}$ sodium azide were spotted at a control location (location B in Fig. 1A).
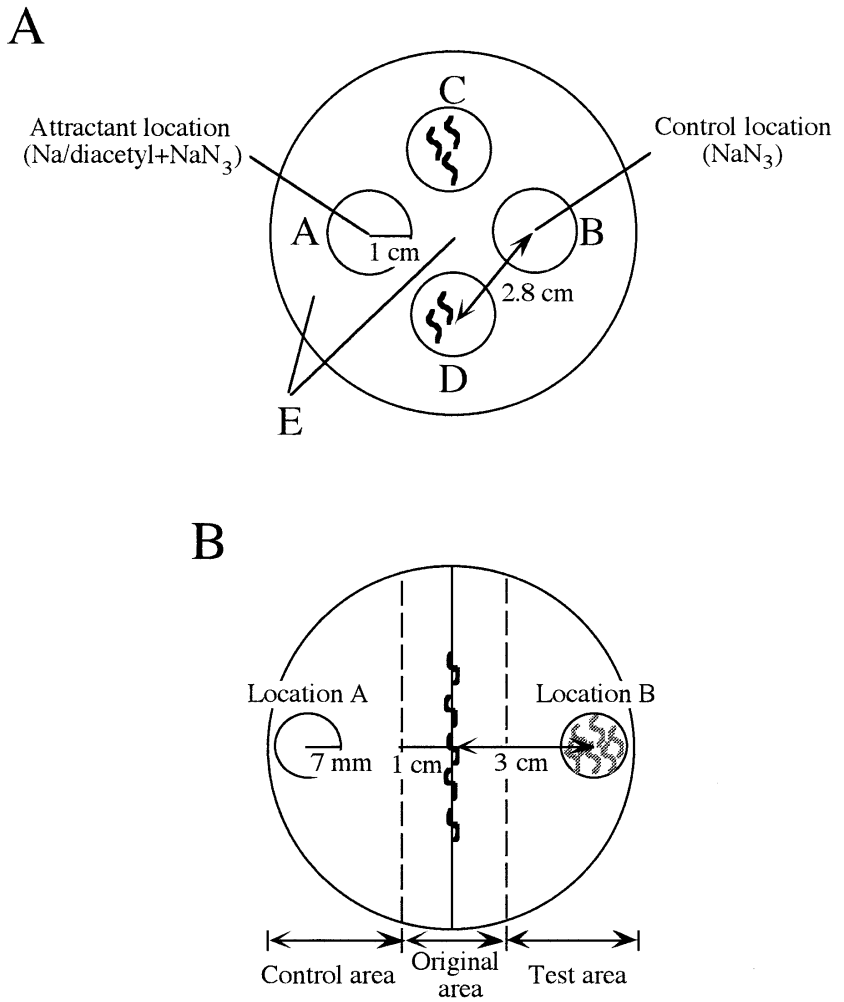

Fig. 1. Configuration of assay plate. (A) Chemotaxis to attractants. Nematodes were placed in two locations ( $C$ and $D)$ equidistant (approximately $2.8 \mathrm{~cm}$ ) from the attractant and control spots (A and B). For the assay of either sodium acetate or diacetyl, $7 \mu \mathrm{l}$ of $1.0 \mathrm{M}$ sodium acetate $(\mathrm{Na})$ or $1.5 \mu \mathrm{l}$ of $0.1 \%$ diacetyl was spotted at the center of the attractant location. To anesthetize the animals, $1 \mu \mathrm{l}$ of $0.5 \mathrm{M}$ sodium azide was spotted at the attractant and control locations. The numbers of animals at both locations ( $A$ and $B$ ) were determined every 10 min over a 90-min period. (B) Assays for substance produced by nematodes. Approximately 30 animals marked with Indian ink were placed at the midline of the assay plate. A 7$\mathrm{mm}$-radius circular plug of agarose containing 100 animals was placed at location $B$ in the test area. The plug in the absence of animals was also placed at location $A$ in the control area. The numbers of animals at the test and control areas were determined for $60 \mathrm{~min}$. 
Nematodes were washed in a 15-ml conical centrifuge tube with buffer ( $1 \mathrm{mM} \mathrm{CaCl}_{2}, 1 \mathrm{mM} \mathrm{MgSO}_{4}, 5 \mathrm{mM} \mathrm{KH}_{2} \mathrm{PO}_{4}$ ( $\mathrm{pH} \mathrm{6.0)}$ ), and $0.05 \%$ Tween 20 ) after removal from the growth plates with bacteria as food, and centrifuged for $20 \mathrm{sec}$ at $600 \mathrm{rpm}$. All the animals were washed three times prior to use. The nematodes were then aspirated from the bottom of the tube using a micropipette ( 3 $\mu \mathrm{l})$ and placed at two locations $(C$ and $D)$ equidistant $(2.8 \mathrm{~cm})$ from the attractant and control spots (Fig. 1A). The animals from both locations were allowed to dry, and the clumped animals were separated from each other by gently touching them with a Kimwipe and then left to move freely on the assay plate for $90 \mathrm{~min}$. The numbers of animals at the attractant and control locations A and B (circle of $1 \mathrm{~cm}$ radius) were determined under a microscope (OLYMPUS SZ40) every $10 \mathrm{~min}$. All experiments were performed during daytime at $20^{\circ} \mathrm{C}$.

To determine the effect of population density at an attractant location on chemotaxis, nematodes that gathered at the attractant location of sodium acetate were removed every $10 \mathrm{~min}$ or at $40 \mathrm{~min}$. In this experiment, we used approximately 100 animals per plate. A control assay, in which the lid of the plate was opened but the nematodes were not removed from the attractant location, was also performed under the condition of a high population density (approximately 100 animals). The total number of animals that gathered and were removed from the attractant location was determined for a 90min period.

The effect of a high population density at an attractant location on chemotaxis was also investigated. Approximately 150 animals were placed at the two original locations and then gathered at the attractant location of $1.0 \mathrm{M}$ sodium acetate or $0.1 \%$ diacetyl for 60 min in advance. When the number of animals at the attractant location were fewer than 100 , some nematodes at the other locations were gently moved to the attractant location using a platinum wire ( $0.1 \mathrm{~mm}$ diameter). Then approximately 30 pellet animals marked with Indian ink were placed at the original locations ( $C$ and $D)$, and their chemotactic responses were observed for $90 \mathrm{~min}$. To mark the assay nematodes, these animals were given food ( $E$. coll) with Indian ink for 3 hours before the assay. No difference in chemotactic response between the marked and nonmarked nematode groups was observed (data not shown).

\section{Assays for substance produced by nematodes}

To examine whether the substance produced by nematodes, i.e., a pheromone, affects the behavior of animals as repellant, the responses of nematodes to $\mathrm{N} 2$ animals and/or daf-22 (m130) mutants of a high population density were tested in the absence of an attractant. First, approximately 100 animals were gathered at the location of diacetyl for $60 \mathrm{~min}$. In this case, $1.3 \mu \mathrm{l}$ of $0.01 \%$ diacetyl was placed at the lid of the plate. To anesthetize the animals, $1 \mu \mathrm{l}$ of $0.5 \mathrm{M}$ sodium azide was spotted on the surface of the plate. Then the lid of the plate was opened, and the plate was left for $60 \mathrm{~min}$ in air to remove an efficacy of diacetyl. After that, a 7-mm-radius circular plug of agarose was removed from another plate (assay plate), and the same size of plug of agarose containing approximately 100 animals was placed in the space where the agarose had been removed (location B in the test area; Fig. 1B). The plug, which was pre-exposed to $0.01 \%$ diacetyl in the absence of nematodes, was also placed at an opposite location (location $A$ in the control area; Fig. 1B). The space between the assay plate and the plug was filled with agarose.

Nematodes marked with Indian ink were washed three times, and approximately 30 animals were placed at the midline of the assay plate (Fig. 1B). The preparation of this experiment took $2 \mathrm{hr}$. When the duration of assay was more than $60 \mathrm{~min}$, some animals came out of anesthesia. Therefore, the numbers of animals at the test and control areas were determined under a microscope for 60 min in this assay (Fig. 1B). Nematodes that remained within $1 \mathrm{~cm}$ from the midline (original area) were not counted, because animals accidentally wandered into either the test and control areas.

\section{Scoring and analysis}

In chemotaxis assays (Fig. 1A), the chemotaxis index was calculated based on the number of animals that gathered at the attractant location (Bargmann et al. 1993). In the assays for the substance produced by nematodes (Fig. 1B), the population index and fraction of animals were also calculated using the following equation:

Chemotaxis index $=($ number of animals at attractant locationnumber of animals at control location) / total number of animals on plate.

Population index $=($ number of animals in test area - number of animals in control area) / total number of animals on plate.

Fraction of animals=number of animals in test area or control area / total number of animals on the plate.

The chemotaxis and population indices showed a positive value when the nematodes elicited the attractive response, while the indices were less than zero when the nematodes showed an avoidance response. The correlation coefficient between the chemotaxis index and the total number of animals on a plate was analyzed using statistical software (Microsoft Excel 2001). The significance of difference in chemotaxis index was statistically analyzed by Student's $t$ test. Values are presented as means \pm S.E.M. A minimum of ten assays were performed for each series of experiments.

\section{RESULTS}

\section{Change in chemotaxis index by population density at attractant location}

To determine the effect of population on chemotactic response to an attractant, the chemotactic indices of N2 animals to a water-soluble attractant (sodium acetate) and a volatile odorant (diacetyl) at various population densities were examined. Under both conditions of low (approximately 30 animals on the assay plate) and high (approximately 100 animals on the assay plate) populations, the chemotaxis index for $1.0 \mathrm{M}$ sodium acetate increased rapidly within $40 \mathrm{~min}$ and then continued to increase gradually during the assay. However, the value under the condition of a high population was significantly lower than that under the condition of a low population $(\mathrm{p}<0.05)$ (Fig. 2A). The chemotaxis indices for sodium acetate under low and high populations at $90 \mathrm{~min}$ were $0.84 \pm 0.03$ and $0.64 \pm 0.05$, respectively. As shown in Fig. $2 \mathrm{~B}$, a negative correlation between the chemotaxis index at $90 \mathrm{~min}$ and the number of animals on the plate was observed $(r=0.57368, p<0.001)$.

Under both conditions of low and high populations, the chemotaxis index for $0.1 \%$ diacetyl increased rapidly within $40 \mathrm{~min}$ and then continued to increase gradually during the 90 min of assay (Fig. 2C). Similar to the response to sodium acetate, the index under a high population was lower than that under a low population, and a significant difference between these indices was observed at $90 \mathrm{~min}(\mathrm{p}<0.05)$. The chemotaxis indices for diacetyl under low and high populations at $90 \mathrm{~min}$ were $0.73 \pm 0.03$ and $0.58 \pm 0.07$, respectively. The chemotaxis index at 90 min showed a significant negative correlation with the number of animals on the plate $(r=0.41927, p<0.001)$ (Fig. 2D).

Under the condition of a high population, the nema- 

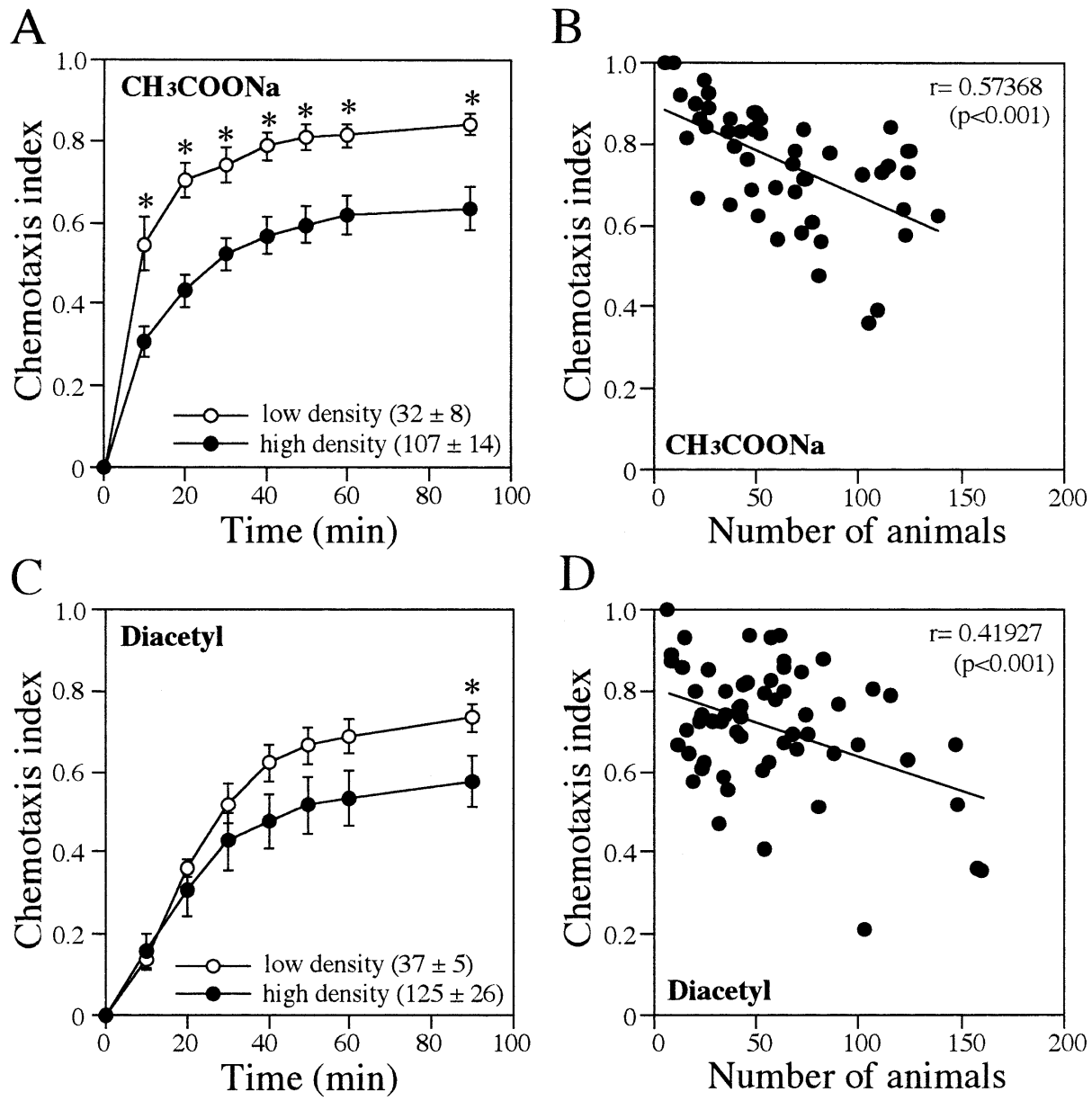

Fig. 2. Chemotactic responses to sodium acetate and diacetyl. (A) Time course of change in average chemotaxis index for sodium acetate.

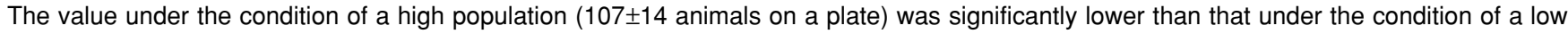
population ( $32 \pm 8$ animals on a plate) $(* \mathrm{p}<0.05)$. (B) Correlation between chemotaxis index for sodium acetate at 90 min and number of animals on assay plate. Chemotaxis index showed a significant negative correlation with the number of animals on the plate $(r=0.57368$, $\mathrm{p}<0.001)$. (C) Time course of change in average chemotaxis index for diacetyl. The value under the condition of a high population (125 \pm 26 animals on a plate) was lower than that under the condition of a low population $(37 \pm 5$ animals on a plate) $(* p<0.05)$. (D) Correlation between chemotaxis index for diacetyl at $90 \mathrm{~min}$ and number of animals on assay plate. Chemotaxis index showed a significant negative correlation with the number of animals $(r=0.41927, \mathrm{p}<0.001)$. Error bars indicate $\mathrm{SEM}(\mathrm{N}=10$ assays $)$.

todes that gathered at the attractant location were removed from the attractant location of sodium acetate every $10 \mathrm{~min}$ or at 40 min during the assay to confirm the effect of population density at the attractant location (Fig. $3 \mathrm{~A})$. When the animals were removed every $10 \mathrm{~min}$ or at $40 \mathrm{~min}$, the chemotaxis indices at 90 min were significantly greater than that for the control assay $(p<0.05)$ (Fig. 3B). These results indicate that an increase in population density at an attractant location affects a chemotactic response, suggesting that attraction to chemicals is regulated by some substances produced by nematodes.

\section{Chemotactic response of daf-22 (m130) mutants}

It is known that daf-22 (m130) mutants show a defective secretion of the pheromone but can sense such a pheromone (Golden and Riddle, 1984; 1985). The chemotactic response of daf-22 (m130) mutants under the condition of low and high population densities was observed. Under both conditions of low and high populations, the chemotaxis index for sodium acetate increased rapidly within $30 \mathrm{~min}$ and then continued to increase gradually during the assay (Fig. $4 A)$. There was no significant difference in the chemotaxis index between the conditions of low and high populations. The chemotaxis indices for sodium acetate at $90 \mathrm{~min}$ under low and high populations were $0.79 \pm 0.04$ and $0.82 \pm 0.04$, respectively. A nonsignificant difference in the chemotaxis index for diacetyl between the conditions of low and high populations was also observed (Fig. 4B). The indices for diacetyl at 90 min under low and high populations were $0.71 \pm 0.04$ and $0.74 \pm 0.04$, respectively.

\section{Effect of pheromone on chemotactic response}

The dauer pheromone of $C$. elegans is used as a measure of population density (Golden and Riddle, 1984). From the results of the above experiments, we have speculated that the attraction to chemicals is regulated by an increase 

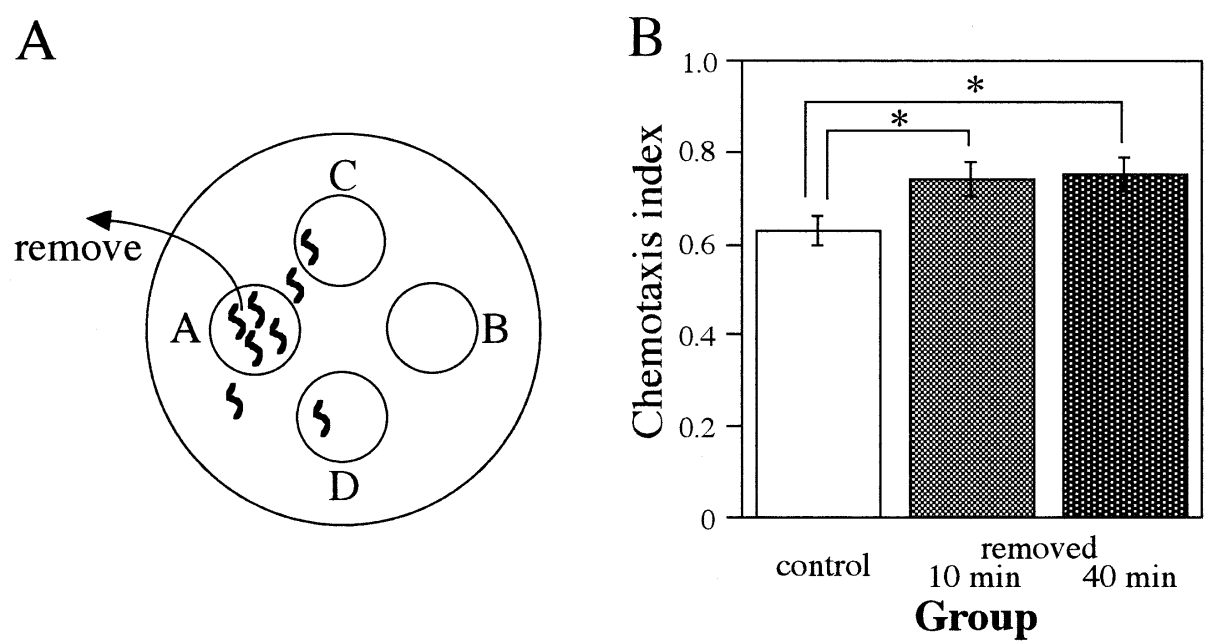

Fig. 3. Effect of population density at attractant location on chemotaxis index for sodium acetate. (A) Scheme of experiment. Under the condition of a high population, the animals that gathered at the attractant location were removed from the attractant location every 10 min or at 40 min during the assay. (B) Change in chemotaxis index at $90 \mathrm{~min}$ among experimental groups. When the nematodes were removed every 10 min or at $40 \mathrm{~min}$, the chemotaxis indices at 90 min were significantly greater than that for the control assay $(* p<0.05)$. In the control assay, the lid of the plate was opened but the animals were not removed from the test location. Note that an increase in population density at an attractant location affects the chemotactic response. Error bars indicate SEM ( $\mathrm{N}=12$ assays).
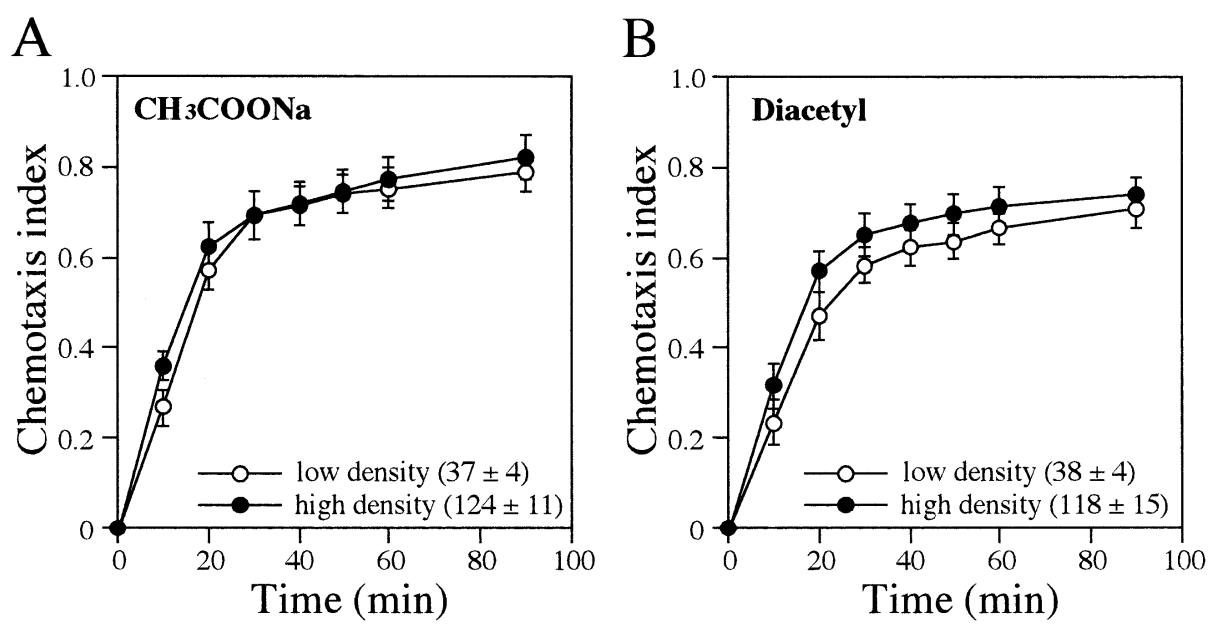

Fig. 4. Time course of change in average chemotaxis index of daf-22 (m130) mutants for sodium acetate (A) and diacetyl (B). Note that there was no significant difference in the chemotaxis index between the conditions of low and high populations regardless of the type of attractant. Error bars indicate SEM ( $\mathrm{N} \geq 11$ assays).

in pheromone concentration at an attractant location. To confirm this possibility, the effect of a high population density at a particular attractant location on chemotaxis was investigated.

When the nematodes were placed at the attractant location in advance, the chemotactic response of the animals at the original location was determined (Fig. 5A). When daf-22 ( $\mathrm{m130}$ ) mutants of a high population density were placed at the attractant location in advance (daf-22/N2 assay), the chemotaxis index of N2 animals for sodium acetate was almost the same as that of the control assay $(-/ \mathrm{Na}$ assay) in which no animals were placed at the attractant location in advance (left panel in Fig. 5B). However, when $\mathrm{N} 2$ animals of a high population density were placed at the attractant location in advance (N2/N2 assay), the chemotaxis index of the animals for sodium acetate was significantly smaller than those of the control (-/N2) and daf-22/N2 assays $(p<0.05)$ (left panel in Fig. 5B). The chemotaxis indices were $0.66 \pm 0.03$ in N2/N2, $0.74 \pm 0.03$ in $-/ \mathrm{N} 2$ and $0.74 \pm 0.03$ in daf-22/N2 assays.

When the $\mathrm{N} 2$ animals of a high population density were placed at the attractant location in advance (N2/daf-22 assay), the chemotaxis index for sodium acetate of daf-22 (m130) mutants was significantly smaller than that of the control (-/daf-22 assay) in which no animals were placed at the attractant location in advance $(p<0.05)$ (right panel in Fig. 5B). The indices were $0.70 \pm 0.03$ in $\mathrm{N} 2 /$ daf-22 and $0.81 \pm 0.03$ in -/daf-22 assays. 


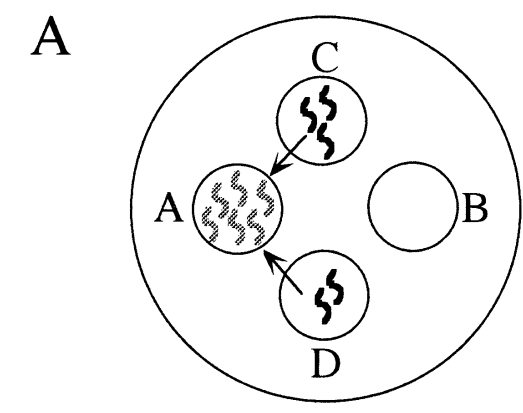

\begin{tabular}{ccc} 
& $\begin{array}{c}\text { location A } \\
\text { (100 animals) }\end{array}$ & $\begin{array}{c}\text { location C, D } \\
\text { (30 animals) }\end{array}$ \\
\hline- / N2 & - & wild type \\
daf-22 / N2 & daf-22 & wild type \\
$\mathrm{N} 2$ / N2 & wild type & wild type \\
$-/$ daf-22 & - & daf-22 \\
N2 / daf-22 & wild type & daf-22 \\
\hline
\end{tabular}

$\mathrm{B}$

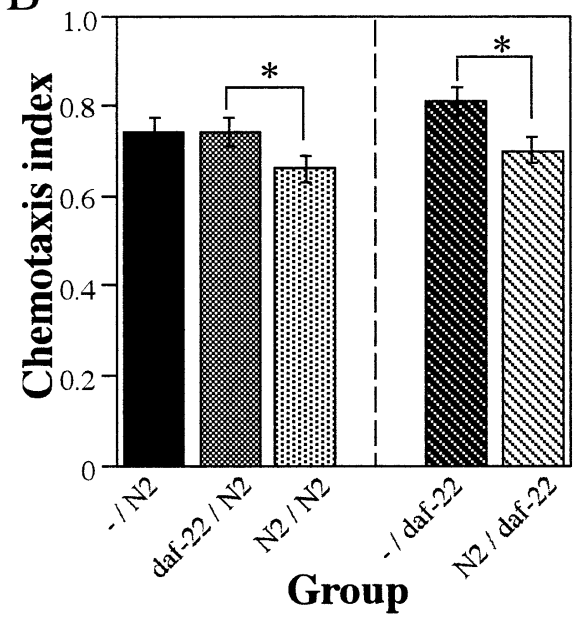

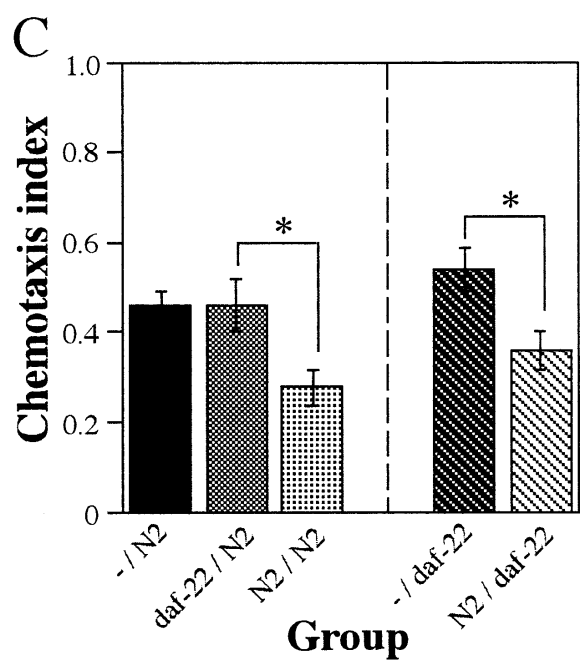

Fig. 5. Effect of a high population density at attractant location on chemotaxis index. (A) Scheme of experiment. Approximately 100 nematodes were placed at the attractant location 60 min before the assay, and then approximately 30 nematodes marked with Indian ink were placed at the original locations ( $C$ and $D)$, and their chemotactic responses were observed for 90 min. (B) Change in chemotaxis index for sodium acetate at $90 \mathrm{~min}$ among experimental groups. (C) Change in chemotaxis index for diacetyl at 90 min among experimental groups. N2 animals gathered at the attractant location, which was the same as that in the case of the control assay in which no animals were placed at the attractant location in advance, even when daf-22 (m130) mutants of a high population density were placed at the attractant area in advance (daf-22/N2 assay). On the other hand, when N2 animals of a high population density were placed at the attractant location in advance (N2/daf22 assay and N2/N2 assay), the chemotaxis index of N2 animals or daf-22 (m130) mutants was significantly smaller than that obtained in the control assay. Error bars indicate SEM ( $\mathrm{N}=16$ assays). $* \mathrm{p}<0.05$.

Similar to the response to sodium acetate, when daf-22 (m130) mutants of a high population density were placed at the attractant location in advance (daf-22/N2 assay), the chemotaxis index of N2 animals for diacetyl was almost the same as that of the control assay (-/N2 assay) in which no animals were placed at the attractant location in advance (left panel in Fig. 5C). In contrast, when N2 animals of a high population density were placed at the attractant area in advance (N2/N2 assay), the chemotaxis index for diacetyl was significantly smaller than those of the control (-/N2) and daf-22/N2 assays $(p<0.05)$ (left panel in Fig. $5 \mathrm{C})$. The indices were $0.46 \pm 0.03$ in $-/ \mathrm{N} 2,0.46 \pm 0.06$ in daf-22/N2 and $0.28 \pm 0.04$ in N2/N2 assays.

When N2 animals of a high population density were placed at the attractant area in advance (N2/daf-22 assay), the chemotaxis index for diacetyl of daf-22 (m130) mutants was significantly smaller than that of the control assay (-/daf22 assay) in which no animals were placed at the attractant 
location in advance $(p<0.05)$ (right panel in Fig. $5 C)$. The chemotaxis indices were $0.36 \pm 0.04$ in N2/daf-22 and $0.54 \pm 0.05$ in -/daf-22 assays.

The chemotaxis indices for diacetyl in above assays were smaller than those of the previous experiments (Figs. $2 \mathrm{C}$ and $4 \mathrm{~B}$ ). In the assays, we spotted diacetyl at an attractant location of an assay plate $1 \mathrm{hr}$ before the start of the assay to ensure a high population density of nematodes at the attractant location in advance, whereas it was spotted shortly before the assay in the previous experiments. Therefore, the difference in the indices was caused by a change in the efficacy of diacetyl at the attractant location.

As described above, daf-22 (m130) mutants cannot secrete but can sense the pheromone. These results suggest that an increase in pheromone concentration at an attractant location inhibits the attractive response of nematodes.

\section{Response to pheromone produced by $\mathrm{N} 2$ animals}

In the above experiments, we investigated the effect of a substance produced by $\mathrm{N} 2$ animals, i.e., a pheromone, on the chemotaxis, and revealed that the chemotactic response of the nematode was inhibited by the pheromone. The question arose as to whether the pheromone is a repellant or not. To clarify this question, we had to directly investigate the effect of the pheromone on animal's behavior in the absence of an attractant. However, it was difficult for us to purify the

A

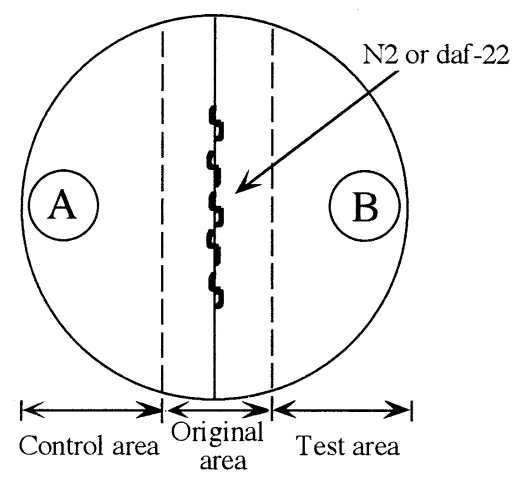

\begin{tabular}{ccc} 
& location A & location B \\
\hline$-: \mathrm{Na}$ & - & sodium acetate \\
$-:$ N2+Na & - & sodium acetate \& wild type \\
$-:-$ & - & - \\
$-:$ daf -22 & - & daf -22 \\
$-:$ N2 & - & wild type \\
daf-22 : N2 & daf-22 & wild type \\
\hline
\end{tabular}

B

C
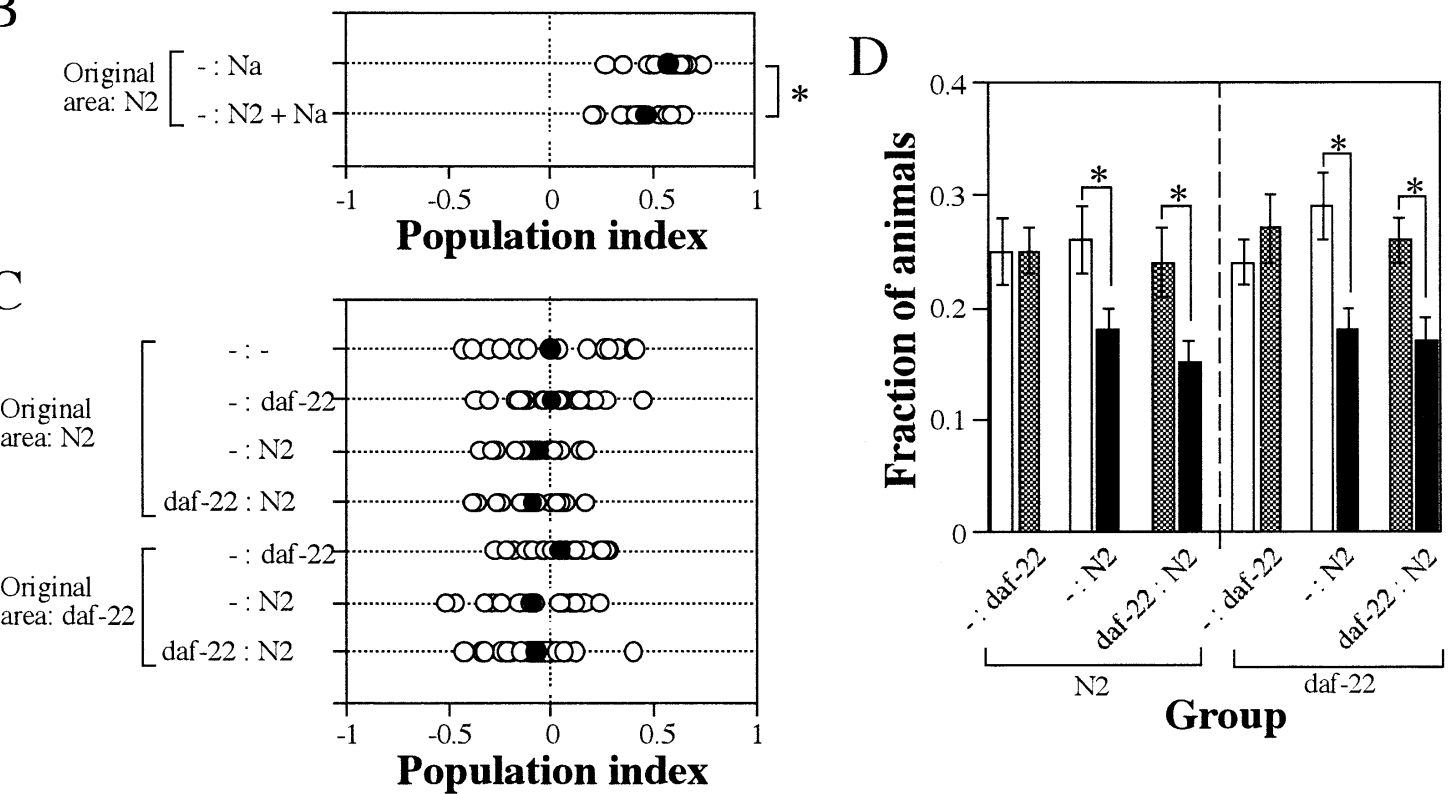

Fig. 6. Effect of pheromone produced by $\mathrm{N} 2$ animals on nematode's behavior. (A) Scheme of experiment. The plug in the absence of animals or containing approximately 100 daf-22 (m130) mutants placed at location A, and the plug containing approximately $100 \mathrm{~N} 2$ animals or daf-22 (m130) mutants was placed at location B. Approximately $30 \mathrm{~N} 2$ animals or daf-22 (m130) mutants marked with Indian ink were placed at the midline of the assay plate. The numbers of animals, which moved from the original area, at the test and control areas were determined for 60 min. (B) Confirmation of efficacy of pheromone on chemotaxis in this experimental procedure. When N2 animals of a high population density were placed at the attractant area in advance (-:N2+Na assay), the population index of $\mathrm{N} 2$ animals for sodium acetate declined in comparison with that for only sodium acetate (-:Na assay). (C) Response to pheromone produced by nematodes. The population indices of $-: \mathrm{N} 2$, -:daf-22 and daf-22:N2 assays were not significantly different from that of the control assay (-:- assay). Open and closed circles are population indices of individual assay and means, respectively. (D) Fraction of animals at the test and control areas. White bars, fraction of animals at the control area; gray bars, fraction of animals at area of daf-22 (m130) mutants of a high population density; black bars, fraction of animals at area of N2 animals of a high population density. Error bars indicate SEM ( $\mathrm{N} \geq 20$ assays). $* \mathrm{p}<0.05$. 
pheromone. Therefore, the responses of nematodes to N2 animals and/or daf-22 (m130) mutants of a high population density were tested in the absence of an attractant using a different assay plate configuration (Figs. 2B and 6A). This configuration has an advantage for determining the effect of the repellant, and has usually been used for repellant assay (Tajima et al, 2001).

We confirmed that the pheromone reduced the attraction to chemicals by this experimental procedure. When N2 animals of a high population density were placed with sodium acetate in advance at location B in the test area (-: $\mathrm{N} 2+\mathrm{Na}$ assay), the chemotaxis index of $\mathrm{N} 2$ animals for sodium acetate was significantly smaller than that for only sodium acetate (-:Na assay) (Fig. 6B). This result indicates that the effect of pheromone produced by $\mathrm{N} 2$ animals was reviewed in this measurement.

When N2 animals were placed in the original area, and daf-22 (m130) mutants of a high population density were placed at location B in the test area (-:daf-22 assay), there was no significant difference in the fraction of N2 animals between the test and control areas (left panel in Fig. 6D). However, when N2 animals of a high population density were placed at location B in the test area (-:N2 assay), the fraction of animals at the test area was significantly smaller than that at the control area in which no animals were placed in advance $(p<0.05)$ (left panel in Fig. 6D). Moreover, when daf-22 (m130) mutants and N2 animals were placed at location $A$ in the control area and at location $B$ in the test area, respectively, (daf-22:N2 assay), the fraction of animals at the test area (side of $\mathrm{N} 2$ animals) was smaller than that at the control area (side of daf-22 (m130) mutants) $(p<0.05)$ (left panel in Fig. 6D). On the other hand, the population indices of -:N2, -:daf-22 and daf-22:N2 assays were not significantly different from that of the control assay (-:- assay) (Fig. 6C), indicating that an avoidance response of nematodes to the pheromone was not observed in the absence of the attractant. This result suggests that the pheromone does not affect the behavior of the nematodes as a strong repellant.

Similar responses were also observed when daf-22 (m130) mutants were placed in the original area. We detected no significant difference in the population index among assay groups of daf-22 (m130) mutants (Fig. 6C). On the other hand, when $\mathrm{N} 2$ animals of a high population density were placed at location $B$ in the test area $(-: \mathrm{N} 2$ assay and daf-22:N2 assay), the fraction of daf-22 (m130) mutants at the test area was significantly smaller than that at the control area (right panel in Fig. 6D). These results suggest that the pheromone produced by $\mathrm{N} 2$ animals inhibits the entrance of the nematodes to an area of N2 animals of a high population density.

\section{DISCUSSION}

In the present study, we investigated the effect of a nematode population on chemotaxis and revealed that the chemotactic response is affected by the nematode population. The chemotaxis index of N2 animals decreased with an increase in the number of animals on the assay plate regardless of the type of attractant, i. e., water-soluble substrate (sodium acetate) or volatile odorant (diacetyl) (Fig. 2). Nuttly et al. (2001) also reported that the time of aversive response to $100 \%$ benzaldehyde for the individually tested animals is shorter than that for the group-tested animals. These observations suggest the existence of interactions between individuals during population assays.

Two possible mechanisms underlying the interactions between individual animals are proposed. One is the suppression of chemical stimulation by mechanical stimulation during population assays. Mechanical stimulation, which is received by animals at the original location, may be enhanced with an increase in the number of animals on a plate due to contact with each other, and may compete with chemical stimulation. However, we rule out this possibility. In spite of the condition of a high population density at the original location, when $\mathrm{N} 2$ animals were removed from the attractant location, the chemotaxis index showed a significant increase in comparison with that for the control assay in which no nematodes were removed from the attractant location (Fig. 3). Moreover, the chemotaxis response of daf22 (m130) mutants under the conditions of a high population at the original location was almost the same as that under the conditions of a low population (Fig. 4). These results indicate that increase in mechanical stimulation by a high population density at the original location does not affect attraction to chemicals.

Another possible mechanism is interaction with substances such as pheromones. We propose the existence of a chemotaxis regulation pheromone (CR-pheromone). daf22 (m130) mutants do not form dauers if crowded and starved, and a pheromone extract prepared from these mutants is not capable of inducing dauer formation in N2 animals (Golden and Riddle, 1985). However, these mutants are capable of forming dauers in response to exogenously supplied pheromones (Ailion and Thomas, 2000). This suggests that daf-22 (m130) mutants are defective in secretion of pheromones including the dauer pheromone but respond normally to the pheromones. On the other hand, wild type N2 animals produce the dauer pheromone and use it to measure population density (Golden and Riddle, 1984). In the present study, when N2 animals of a high population density were placed at the attractant location in advance, the chemotaxis indices of both $\mathrm{N} 2$ animals and daf-22 (m130) mutants were significantly lower than those in the case of the control in which no animals were placed at the attractant location in advance (Fig. 5). In contrast, both N2 animals and daf-22 (m130) mutants could gather at the attractant location, which was the same as in the control assay in which no animals were placed at the attractant location in advance, even when daf-22 (m130) mutants of a high population density were placed at the attractant location in advance (Figs. 4 and 5). Because of the defective 
CR-pheromone secretion by daf-22 (m130) mutants, the concentration of pheromone at the attractant location did not increase. Therefore, the nematodes could gather at the attractant location. The CR-pheromone is one of the substances which is produced by N2 animals but not daf-22 (m130) mutants, although it is not clear that the CR-pheromone is the same substance as the dauer pheromone.

In the absence of an attractant, we observed a decline in the fraction of animals at the area of N2 animals of a high population density, although the nematodes were not influenced by daf-22 ( $m 130$ ) mutants of a high population density (Fig. 6D). On the other hand, there was no significant difference in population indices among assays for N2 animals, daf-22 (m130) mutants and the control (Fig. 6C). These results imply two opposite possibilities. The first possibility is that the CR-pheromone produced by N2 animals is a repellant, but the effect of the CR-pheromone as a repellant was not detected in the experiment because of a larger test area in comparison with the attractant location of chemotaxis assay. However, in this assay for the pheromone, we confirmed that the population index of $\mathrm{N} 2$ animals for sodium acetate, which was calculated using the number of animals in the test and control areas, was reduced by CRpheromone (Fig. 6B). This indicates that the CR-pheromone has an effect on an animal's behavior in the test area of this assay. The second possibility is that the CR-pheromone is not a repellant, but inhibits the entrance of the nematodes to a high-pheromone-concentration area. In this experiment, we revealed that the $\mathrm{CR}$-pheromone was not a strong repellant. However, we could not conclude whether the CR-pheromone is a weak repellant or not. Further experiments, such as pheromone purification or daf-22 cloning, are required to clarify the mechanisms of the pheromonal regulation of chemotaxis.

We revealed that attraction to chemicals is inhibited by an increase in the CR-pheromone concentration at an attractant location due to an increase in population density. Why does $C$. elegans need the mechanism of population density regulation at an attractant location? $C$. elegans can chemotax toward the peak of a gradient of a number of small molecules, such as $\mathrm{Na}^{+}, \mathrm{K}^{+}, \mathrm{Cl}^{-}$, cAMP, lysine and cysteine, which are produced by food bacteria (Ward, 1973; Dusenbery, 1974). The signal of these substances indicates the existence of food for the nematode, and the chemotactic behavior of $C$. elegans may arise as a result of food detection. When many animals gather at a food source, the volume of food that each individual receives is smaller, and the decrease in the amount of food is faster than that when only a few animals gather at food location. Pheromones are produced by one animal to communicate with each other and initiate a range of physiological responses. For individual nematodes to survive, the mechanism of population density regulation at an attractant (food) location by pheromone release may have developed during their evolution.

\section{ACKNOWLEDGMENTS}

The helpful suggestion of Dr Tokumitsu Wakabayashi is gratefully acknowledged. The nematode strains were provided by the Caenorhabditis Genetics Center. This work was partially supported by a grant for Scientific Research from the Japanese Ministry of Education, Culture, Sports, Science and Technology to T. M.

\section{REFERENCES}

Ailion M, Thomas JH (2000) Dauer formation induced by high temperatures in Caenorhabditis elegans. Genetics 156: 1047-1067

Albertson DG, Thomson JN (1976) The pharynx of C. elegans. Philos Trans R Soc Lond B Biol Sci 275: 299-325

Andrew PA, Nicholas WL (1976) Effect of bacteria on dispersal of Caenorhabditis elegans (Rhabditiae). Nematologica 22: 451461

Bargmann Cl, Horvitz HR (1991) Chemosensory neurons with overlapping functions direct chemotaxis to multiple chemicals in $C$. elegans. Neuron 7: 729-742

Bargmann Cl, Hartwieg E, Horvitz HR (1993) Odorant-selective genes and neurons mediate olfaction in $C$. elegans. Cell 74: 515-527

Brenner S (1974) The genetics of Caenorhabditis elegans. Genetics 7: 71-94

Chalfie M, Sulston JE, White JG, Southgate E, Thomson JN, Brenner $S$ (1985) The neuronal circuit for touch sensitivity in Caenorhabditis elegans. J Neurosci 5: 956-964

Dusenbery DB (1974) Analysis of chemotaxis in the nematode Caenorhabditis elegans by countercurrent separation. J Exp Zool 188: $41-47$

Golden JW, Riddle DL (1984) The Caenorhabditis elegans dauer larva: Developmental effects of pheromone, food and temperature. Dev Biol 102: 368-378

Golden JW, Riddle DL (1985) A gene affecting production of the Caenorhabditis elegans dauer-inducing pheromone. Mol Gen Genet 198: 534-536

Hilliard MA, Bargmann Cl, Bazzicalupo P (2002) C. elegans responds to chemical repellents by integrating sensory inputs from the head and the tail. Current Biol 12: 730-734

Matsuura T, Oikawa T, Wakabayashi T, Shingai R (2004a) Effect of simultaneous presentation of multiple attractants on chemotactic response of the nematode Caenorhabditis elegans. Neurosci Res 48: 419-429

Matsuura T, Sato T, Wakabayashi T, Shingai R (2004b) Effect of population density on chemotactic response in the nematode Caenorhabditis elegans. Zoo Sci 21: 1321

Nuttley WM, Harbinder S, van der Kooy D (2001) Regulation of distinct attractive and aversive mechanisms mediating benzaldehyde chemotaxis in Caenorhabditis elegans. Learn Mem 8: 170-181

Riddle DL, Albert PS (1997) Genetic and environmental regulation of dauer larva development. In " $C$. elegans II" Ed by DL Riddle, T Blumenthal, BJ Meter, JR Priess, Cold Spring Harbor Laboratory Press, New York, pp 739-768

Tajima T, Waranabe N, Kogawa Y, Takiguchi N, Kato J, Ikeda T, kuroda A, Ohtake $\mathrm{H}$ (2001) Chemotaxis of the nematode Caenorhabditis elegans toward cycloheximide and quinine hydrochloride. J Biosci Bioeng 91: 322-324

Ward S (1973) Chemotaxis in the nematode Caenorhabditis elegans: identification of attractants and analysis of the response by use of mutants. Proc Natl Acad Sci USA 70: 817-821

Wicks SR, Rankin CH (1995) Integration of mechanosensory stimuli in Caenorhabditis elegans. J Neurosci 15: 2434-2444 